\title{
Extending Peano derivatives: necessary and sufficient conditions
}

\author{
by
}

Hans Volkmer (Milwaukee, Wisc.)

\begin{abstract}
The paper treats functions which are defined on closed subsets of $[0,1]$ and which are $k$ times Peano differentiable. A necessary and sufficient condition is given for the existence of a $k$ times Peano differentiable extension of such a function to [0,1]. Several applications of the result are presented. In particular, functions defined on symmetric perfect sets are studied.
\end{abstract}

1. Introduction. Let $P$ be a closed subset of $[0,1]$, and let $f: P \rightarrow \mathbb{R}$ be a given real-valued function defined on $P$. Let $k$ be a positive integer. We say that $f$ is $k$ times Peano differentiable at $x \in P$ relative to $P$ with Peano derivatives $f_{(1)}(x), \ldots, f_{(k)}(x)$ if we can write $\left(f_{(0)}:=f\right)$

$$
f(x+h)=\sum_{j=0}^{k} f_{(j)}(x) \frac{h^{j}}{j !}+\varepsilon(x, h) \frac{h^{k}}{k !}
$$

with

$$
\varepsilon(x, h) \rightarrow 0 \quad \text { as } 0 \neq h \rightarrow 0, x+h \in P .
$$

This condition is empty if $x$ is an isolated point of $P$. At an isolated point the Peano derivatives $f_{(1)}(x), \ldots, f_{(k)}(x)$ are arbitrarily assigned. If $f$ is $k$ times Peano differentiable at every point $x \in P$, then we say that $f$ is $k$ times Peano differentiable on $P$ relative to $P$. If $P$ is perfect, this definition is due to Denjoy [4, p. 280]. The extension to closed sets was given by Fejzić, Mařík and Weil [7].

Let $f: P \rightarrow \mathbb{R}$ be $k$ times Peano differentiable on $P$ relative to $P$ with Peano derivatives $f_{(1)}, \ldots, f_{(k)}$. In this paper we deal with the following question: does there exist a function $F:[0,1] \rightarrow \mathbb{R}$ which is $k$ times Peano differentiable on $[0,1]$ and has the property that $F(x)=f(x)$ and $F_{(j)}(x)=$

1991 Mathematics Subject Classification: Primary 26A24. 
$f_{(j)}(x)$ for all $x \in P$ and all $j=1, \ldots, k$ ? We will call such a function $F$ a $k$-extension of $f$ for short.

This question was raised in the very interesting papers $[2,7]$ which inspired the present paper. It was shown in [7] that 1-extensions always exist but examples of Buczolich [1] and Denjoy [4] show that, for every $k \geq 2$, there are $k$ times Peano differentiable functions which do not admit a $k$-extension. A more general class of such examples is presented in Section 4 of the present paper.

The main result of this paper is Corollary 3.10 of Theorem 3.2 which gives a necessary and sufficient condition for the existence of $k$-extensions. The necessity of the condition is known from [7, Cor. 4.8]. We recall this important theorem in Section 2. In Corollary 3.8 we prove that a $k$ times Peano differentiable function $f: P \rightarrow \mathbb{R}$ admits a $k$-extension if and only if its restriction to the perfect kernel of the boundary of $P$ admits a $k$-extension.

As in [7] we say that a closed subset $P$ of $[0,1]$ belongs to the class $\mathbf{P}_{k}$ if every $k$ times Peano differentiable function $f: P \rightarrow \mathbb{R}$ admits a $k$-extension. Corollary 3.9 establishes that every closed set with countable boundary belongs to $\mathbf{P}_{k}$.

In Section 4 we investigate the problem whether a given symmetric perfect set specified by a sequence $\left\{\varepsilon_{n}\right\}$ belongs to $\mathbf{P}_{k}$. For many sequences we solve the problem but one case is still open.

2. A property of Peano derivatives. Let $H$ be a perfect subset of $[0,1]$. We say that $H$ is of finite Denjoy index [3, p. 138], [7, p. 392] if there exist two constants $\theta>0$ and $\beta>1$ such that, for every $x \in H$, there is a real sequence $h_{n}, n \in \mathbb{N}$, such that $0 \neq h_{n} \rightarrow 0$ as $n \rightarrow \infty, x+h_{n} \in H$ for $n \in \mathbb{N},\left|h_{1}\right| \geq \theta$, and

$$
1<\left|h_{n}\right| /\left|h_{n+1}\right| \leq \beta \quad \text { for all } n \in \mathbb{N} .
$$

The following theorem will be used in Section 3.

Theorem 2.1. Let $H$ be a perfect subset of $[0,1]$ of finite Denjoy index. Let $f: H \rightarrow \mathbb{R}$ be $k$ times Peano differentiable on $H$ relative to $H$ with Peano derivatives $f_{(1)}, \ldots, f_{(k)}$. Let $P$ be a perfect subset of $H$. Then there is a dense open subset $E$ of $P$ such that, for each $x \in E$ and $p=1, \ldots, k-1$, $f_{(p)}$ is $k-p$ times Peano differentiable at $x$ relative to $P$ with Peano derivatives $f_{(p+1)}(x), \ldots, f_{(k)}(x)$.

Theorem 2.1 is related to a result of Denjoy [4, p. 293] (which is given without proof), namely that the set $E$ is only residual (complement of a set of first category). Theorem 2.1 is proved for $H=[0,1]$ in [6, Thm. 1.1.20]. In $[7$, Cor. 4.8] an extension theorem is used to generalize it to the case 
where $H$ is of finite Denjoy index. The author has found a more direct proof of Theorem 2.1 that is omitted here. It is of interest to have such a proof because we will show that Theorem 2.1 can be used to prove the extension theorem (Corollary 3.11).

Theorem 2.1 with $H=P=[0,1]$ shows that every function $f:[0,1] \rightarrow \mathbb{R}$ which is $k$ times Peano differentiable on $[0,1]$ is $k$ times differentiable on a dense open subset of $[0,1]$. This was proved by Oliver $[8]$ in a different way.

3. A necessary and sufficient condition. The following lemma shows that we can assume without loss of generality that $P$ is nowhere dense when we study the extension problem.

Lemma 3.1. Let $P$ be a closed subset of $[0,1]$, and let $f: P \rightarrow \mathbb{R}$ be $k$ times Peano differentiable on $P$ relative to $P$. If $f$ restricted to the topological boundary $\partial P$ of $P$ has a $k$-extension, then so does $f$.

Proof. Let $G$ be a $k$-extension of $f \mid \partial P$. The function $h:=f-G$ is $k$ times Peano differentiable on $P$ relative to $P$, and it vanishes together with its first $k$ Peano derivatives on $\partial P$. Define $H:[0,1] \rightarrow \mathbb{R}$ by $H(x)=h(x)$ for $x \in P$ and $H(x)=0$ for $x \notin P$. Then $H$ is a $k$-extension of $h$. Now $G+H$ is a $k$-extension of $f$.

Let $P$ be a nowhere dense closed subset of $[0,1]$, and let $f: P \rightarrow \mathbb{R}$ be $k$ times Peano differentiable on $P$ relative to $P$. Let $R(f, P)$ be the set of all $x \in P$ for which there exists an open interval $(a, b)$ with $a<x<b$ and $a, b \notin P$ such that $f \mid(a, b) \cap P$ has a $k$-extension. Note that $R(f, P)$ is open relative to $P$ and contains every isolated point of $P$. We also set $Q(f, P):=P-R(f, P)$. This is a closed subset of $P$.

Our goal is to prove the following theorem.

THEOREM 3.2. Let $P$ be a closed nowhere dense subset of $[0,1]$, and let $f: P \rightarrow \mathbb{R}$ be $k$ times Peano differentiable on $P$ relative to $P$. If $f$ satisfies the condition:

(3.1) for every nonempty closed subset $P_{0}$ of $P, R\left(f, P_{0}\right)$ is nonempty,

then $f$ admits a k-extension.

For the proof a series of lemmas will be needed.

Lemma 3.3. Let $P$ be a closed subset of $[0,1]$. Let $f: P \rightarrow \mathbb{R}$ be $k$ times Peano differentiable on $P$ relative to $P$. Suppose there is a $k$-extension $F:[0,1] \rightarrow \mathbb{R}$ of $f$. For every open interval $I$ containing $P$ and every $\varepsilon>0$, there is another $k$-extension $H:[0,1] \rightarrow \mathbb{R}$ of $f$ such that

$$
\max _{x \in[0,1]}|H(x)| \leq \max _{x \in P}|f(x)|+\varepsilon
$$


and

$$
H(x)=0 \quad \text { for all } x \text { outside } I .
$$

Proof. Let $A:=\max _{x \in P}|f(x)|$. Define a function $G:[0,1] \rightarrow \mathbb{R}$ by $G(x)=F(x)$ if $|F(x)| \leq A+\varepsilon, G(x)=A+\varepsilon$ if $F(x)>A+\varepsilon$ and $G(x)=-A-\varepsilon$ if $F(x)<-A-\varepsilon$. Then $G$ might not be $k$ times Peano differentiable on $[0,1]$ any more but $G$ agrees with $F$ in a neighborhood of each $x \in P$. Inspection of the proof of Lemma 4.6 of [7] shows that $G$ can be "smoothened" to a function $H$ in such a way that it becomes a $k$-extension of $f$ and still $|H(x)| \leq A+\varepsilon$ for all $x \in[0,1]$. It is clear that we can change $H$ so that $H$ vanishes outside $I$ without destroying condition (3.2).

Lemma 3.4. Let $P$ be a closed nowhere dense subset of $[0,1]$, and let $f: P \rightarrow \mathbb{R}$ be $k$ times Peano differentiable on $P$ relative to $P$. Let $A$ be a compact subset of $R(f, P)$. Then $f \mid A$ admits a k-extension.

Proof. For every $x \in A$, there is an open interval $I$ containing $x$ whose endpoints are not in $P$ such that $f \mid I \cap A$ admits a $k$-extension. By compactness of $A$, finitely many of these intervals, say $I_{1}, \ldots, I_{n}$, cover $A$. We can also assume that these intervals are pairwise disjoint. By Lemma 3.3, for every $j=1, \ldots, n$, there is a $k$-extension $F_{j}$ of $f \mid I_{j} \cap A$ which vanishes outside $I_{j}$. Then $F_{1}+\ldots+F_{n}$ is a $k$-extension of $f \mid A$.

Lemma 3.5. Let $P$ be a closed nowhere dense subset of $[0,1]$. Let $Q$ be a nonempty closed subset of $P$. Then there exists a countable collection of open intervals $I_{n}$ which has the following properties:

(i) the $I_{n}$ are pairwise disjoint, disjoint from $Q$ and $P_{n}:=I_{n} \cap P$ is nonempty;

(ii) the length $\left|I_{n}\right|$ of $I_{n}$ is less than the distance $\operatorname{dist}\left(I_{n}, Q\right)$ from $I_{n}$ to $Q$;

(iii) the endpoints of $I_{n}$ are not in $P$ so that $P_{n}$ is closed;

(iv) $P-Q=\bigcup_{n} P_{n}$.

Proof. Consider a complementary interval $(a, b)$ of $Q$. Since $P$ is nowhere dense, it is easy to find points $c_{n}, n \in \mathbb{Z}$, which are not in $P$ such that $a<\ldots<c_{-1}<c_{0}<c_{1}<\ldots<b, c_{n} \rightarrow a$ as $n \rightarrow-\infty, c_{n} \rightarrow b$ as $n \rightarrow \infty$ and $\operatorname{dist}\left(\left(c_{n}, c_{n+1}\right), Q\right)>\left|c_{n}-c_{n+1}\right|$ for all $n$. Then let $I_{n}=\left(c_{n}, c_{n+1}\right)$. If we do this for every complementary interval, the collection of all the $I_{n}$ that meet $P$ has the desired properties.

Lemma 3.6. Let $P$ be a closed nowhere dense subset of $[0,1]$, and let $f: P \rightarrow \mathbb{R}$ be $k$ times Peano differentiable on $P$ relative to $P$. Suppose that, for all $x \in Q(f, P)$,

$$
f(x)=f_{(1)}(x)=\ldots=f_{(k)}(x)=0 .
$$

Then $f$ admits a k-extension. 
Proof. If $Q:=Q(f, P)$ is empty, then the conclusion follows from Lemma 3.4 with $A=P$. So let $Q$ be nonempty. By Lemma 3.5, there are countably many open intervals $I_{n}$ having the properties (i) through (iv) as given in the lemma. Let $P_{n}:=P \cap I_{n}$. Since $P_{n} \cap Q=\emptyset$, Lemmas 3.3 and 3.4 tell us that, for every $n$, there is $F_{n}:[0,1] \rightarrow \mathbb{R}$ such that

(a) $F_{n}$ is $k$ times Peano differentiable on $[0,1]$;

(b) $\left(F_{n}\right)_{(j)}(x)=f_{(j)}(x)$ for all $x \in P_{n}$ and all $j=0, \ldots, k$;

(c) $F_{n}$ has support in $I_{n}$;

(d) $\left|F_{n}(x)\right| \leq \max _{y \in P_{n}}|f(y)|+\operatorname{dist}\left(I_{n}, Q\right)^{k+1}$ for all $x$.

Define $F:[0,1] \rightarrow \mathbb{R}$ by

$$
F(x):=\sum_{n} F_{n}(x)
$$

This is a well-defined function because the supports of the $F_{n}$ are pairwise disjoint. We now show that $F$ is a $k$-extension of $f$. Each $x \in[0,1]-Q$ has a neighborhood which meets only finitely many supports of the $F_{n}$. This proves that $F$ is $k$ times Peano differentiable at each $x \in[0,1]-Q$. If $x \in P-Q$, then there is $n$ such that $x \in P_{n}$ and $F$ agrees with $F_{n}$ in $I_{n}$. Thus $F_{(j)}(x)=f_{(j)}(x)$ for all $j=0, \ldots, k$.

By (3.3), all what is left to show is that $F(x) /(x-b)^{k} \rightarrow 0$ as $x \rightarrow b$ for every $b \in Q$. Let $b \in Q, \varepsilon>0$. By assumption, there is $0<\delta<\varepsilon$ such that

$$
|y-b|<\delta, y \in P \Rightarrow|f(y)| \leq \varepsilon|y-b|^{k} .
$$

Let $x \in[0,1]$ with $|x-b|<\delta / 2$. Since there is nothing to prove if $F(x)=0$, let $x \in I_{n}$ for some $n$. So

$$
\left|I_{n}\right| \leq \operatorname{dist}\left(I_{n}, Q\right) \leq|x-b| .
$$

If $y \in P_{n}$, then

$$
|y-b| \leq|y-x|+|x-b| \leq\left|I_{n}\right|+|x-b| \leq 2|x-b|<\delta .
$$

By (3.4), $|f(y)| \leq \varepsilon|y-b|^{k} \leq \varepsilon 2^{k}|x-b|^{k}$. By (d) and (3.5),

$$
|F(x)| \leq \varepsilon 2^{k}|x-b|^{k}+|x-b|^{k+1} \leq \varepsilon\left(2^{k}+1\right)|x-b|^{k} .
$$

Since this is true for all $x$ with $|x-b|<\delta / 2$, the conclusion follows.

Let $P$ be a closed nowhere dense subset of $[0,1]$, and let $f: P \rightarrow \mathbb{R}$ be $k$ times Peano differentiable on $P$ relative to $P$. By transfinite induction, for every ordinal $\alpha$, we define a closed subset $T_{\alpha}=T_{\alpha}(f, P)$ of $P$ as follows:

(i) if $\alpha=0$, then $T_{0}:=P$;

(ii) if $\alpha=\beta+1$, then $T_{\alpha}:=Q\left(f, T_{\beta}\right)$;

(iii) if $\alpha$ is a limit number, then $T_{\alpha}:=\cap_{\beta<\alpha} T_{\beta}$.

Clearly, we have $T_{\beta} \subset T_{\alpha}$ (with equality allowed) whenever $\alpha<\beta$. Under condition (3.1), $T_{\beta}$ is a proper subset of $T_{\alpha}$ whenever $\alpha<\beta$ and $T_{\alpha}$ 
is nonempty. In this case the Cantor-Baire stationary principle implies that there is a smallest ordinal $\mu=\mu(f, P)$ in the first or second number class for which $T_{\mu}=\emptyset$. We will use transfinite induction on $\mu$ in order to construct a $k$-extension of $f$. Let us first use an ordinary induction.

Lemma 3.7. Let $P$ be a closed nowhere dense subset of $[0,1]$, and let $f: P \rightarrow \mathbb{R}$ be $k$ times Peano differentiable on $P$ relative to $P$. Assume that there is a positive integer $n$ such that $T_{n}=\emptyset$. Then $f$ admits a $k$-extension.

Proof. The proof is by induction on $n$. If $n=1$, then we are done by Lemma 3.4. Assume that the statement of the lemma is true for $n-1$ in place of $n$, and let $P$ and $f$ be given with $T_{n}(f, P)=\emptyset$. Define $Q:=Q(f, P)$. Then $Q$ is a closed subset of $[0,1]$ with $T_{n-1}(f, Q)=\emptyset$. By induction hypothesis, there is a function $G:[0,1] \rightarrow \mathbb{R}$ which is $k$ times Peano differentiable on $[0,1]$ and $G_{(j)}(x)=f_{(j)}(x)$ for all $x \in Q$ and $j=0, \ldots, k$. The function $f-G$ is $k$ times Peano differentiable on $P$ relative to $P$. This function together with its first $k$ Peano derivatives vanishes on $Q$. Note that $Q(f, P)=$ $Q(f-G, P)$. By Lemma 3.6, there is a function $H:[0,1] \rightarrow \mathbb{R}$ which is $k$ times Peano differentiable on $[0,1]$ and $H_{(j)}(x)=f_{(j)}(x)-G_{(j)}(x)$ for all $x \in P$ and $j=0, \ldots, k$. Now $F:=G+H$ is a $k$-extension of $f$.

We are now in a position to prove Theorem 3.2.

Proof of Theorem 3.2. Let $\mu=\mu(f, P)$ be the smallest ordinal (of the first or second number class) such that $T_{\mu}(f, P)=\emptyset$. We prove the theorem by transfinite induction on $\mu(f, P)$. We have already shown in Lemma 3.7 that the theorem is true if $\mu(f, P)$ is finite. Assume now that the theorem is true if $\mu(f, P)<\gamma$ where $\gamma$ is a given ordinal in the second number class. Let $P$ be a closed nowhere dense subset of $[0,1]$, and let $f: P \rightarrow \mathbb{R}$ be $k$ times Peano differentiable on $P$ relative to $P$ with $\mu(f, P)=\gamma$. We have to show that $f$ admits a $k$-extension. The ordinal $\gamma$ cannot be a limit number. So $\gamma$ is of the form $\gamma=\beta+m$, where $\beta$ is a limit number and $m$ is a positive integer. Let

$$
S:=T_{\beta}=\bigcap_{\alpha<\beta} T_{\alpha}
$$

Since $T_{m}(f, S)=\emptyset$ we know from Lemma 3.7 that $f \mid S$ has a $k$-extension $G$. Define $h(x):=f(x)-G(x)$ for $x \in P$. Note that $T_{\alpha}(f, P)=T_{\alpha}(h, P)$ for all ordinals $\alpha$, and

$$
h(x)=h_{(1)}(x)=\ldots=h_{(k)}(x)=0 \quad \text { for all } x \in S .
$$

Let $x$ be in $P-S$. Then there is an ordinal $\alpha<\beta$ such that $x \notin T_{\alpha}$. Choose an open interval $(a, b)$ disjoint from $T_{\alpha}$ containing $x$ and such that $a, b \notin P$. Then $P_{0}:=P \cap(a, b)$ is disjoint from $T_{\alpha}$. Since $T_{\alpha}\left(h, P_{0}\right)$ is a subset of both $T_{\alpha}=T_{\alpha}(h, P)$ and $P_{0}, T_{\alpha}\left(h, P_{0}\right)$ is empty. By induction hypothesis, $h \mid P_{0}$ 
admits a $k$-extension which implies $x \in R(h, P)$. Since $x$ was arbitrary in $P-S$, we see that $P-S$ is contained in $R(h, P)$ and so $Q(h, P)$ is a subset of $S$. By Lemma 3.6 and (3.6), $h$ admits a $k$-extension $H$. Then $G+H$ is a $k$-extension of $f$.

We now draw some conclusions from Theorem 3.2.

Corollary 3.8. Let $P$ be a closed subset of $[0,1]$, and let $f: P \rightarrow \mathbb{R}$ be $k$ times Peano differentiable on $P$ relative to $P$. Let $\partial P=A \cup B$ be the (unique) decomposition of $\partial P$ into a perfect (or empty) set $A$ and an at most countable set $B$. If $f \mid A$ admits a $k$-extension, then so does $f$.

Proof. We verify that $f \mid \partial P$ satisfies condition (3.1). Let $P_{0}$ be a closed nonempty subset of $\partial P$. If $P_{0}$ has an isolated point, then this point is in $R\left(f, P_{0}\right)$ and $R\left(f, P_{0}\right)$ is nonempty. If $P_{0}$ does not have an isolated point, then $P_{0}$ is perfect and it is a subset of $A$. Since $f \mid A$ has a $k$-extension, this implies $R\left(f, P_{0}\right)=P_{0}$. So condition (3.1) is satisfied, and the conclusion follows from Lemma 3.1 and Theorem 3.2.

Corollary 3.8 shows that it is sufficient to consider nowhere dense perfect sets $P$ when we investigate the extension problem.

Corollary 3.9. Let $P$ be a closed subset of $[0,1]$ with the property that $\partial P$ is countable. Then $P$ belongs to the class $\mathbf{P}_{k}$.

We now obtain a necessary and sufficient condition for the existence of $k$-extensions.

Corollary 3.10. Let $P$ be a closed subset of $[0,1]$, and let $f: P \rightarrow \mathbb{R}$ be $k$ times Peano differentiable on $P$ relative to $P$ with Peano derivatives $f_{(1)}, \ldots, f_{(k)}$. Then there exists a $k$-extension of $f$ if and only if the following condition holds: in every perfect subset $P_{0}$ of $\partial P$ there exists a point $x$ such that, for all $y$ in a neighborhood $I$ of $x$ relative to $P_{0}$ and all $p=$ $1, \ldots, k-1, f_{(p)}$ is $k-p$ times Peano differentiable at $y$ relative to $P_{0}$ with Peano derivatives $f_{(p+1)}(y), \ldots, f_{(k)}(y)$.

Proof. By Theorem 2.1 with $H=[0,1]$, the condition is necessary for the existence of a $k$-extension of $f$. Now let the condition be satisfied. In order to show that $f$ admits a $k$-extension it is enough to verify condition (3.1) for $f \mid \partial P$ (by Lemma 3.1 and Theorem 3.2). Let $P_{0}$ be a perfect subset of $\partial P$. By assumption, there is $x \in P_{0}$ and an open interval $I$ containing $x$ whose endpoints do not lie in $P$ such that for all $y \in I \cap P_{0}$ and all $p=1, \ldots, k-1, f_{(p)}$ is $k-p$ times Peano differentiable at $y$ relative to $P_{0}$ with Peano derivatives $f_{(p+1)}(y), \ldots, f_{(k)}(y)$. By [7, Theorem 3.3], this implies that $f \mid I \cap P_{0}$ admits a $k$-extension.

By combining Theorem 2.1 with Corollary 3.10 we obtain a new proof of the main result of [7]. 
Corollary 3.11. Every perfect subset of $[0,1]$ which has finite Denjoy index belongs to $\mathbf{P}_{k}$.

4. Extension of functions defined on symmetric perfect sets. Let $\lambda_{n}, n \in \mathbb{N}$, be a given sequence of positive numbers with $\sum_{n=1}^{\infty} \lambda_{n}=1$. We assume that

$$
\mu_{n}:=\sum_{m=n+1}^{\infty} \lambda_{m}<\lambda_{n} \quad \text { for all } n \in \mathbb{N} .
$$

Let $P$ be the set of all finite or infinite subsums of the series $\sum_{n} \lambda_{n}$ :

$$
P:=\left\{\sum_{n \in A} \lambda_{n}: A \in \mathbf{P}(\mathbb{N})\right\},
$$

where $\mathbf{P}(\mathbb{N})$ denotes the power set of $\mathbb{N}$. The empty sum is defined as 0 .

Let $T: \mathbf{P}(\mathbb{N}) \rightarrow P$ be the map defined by $T(A):=\sum_{n \in A} \lambda_{n}$. Then $T$ is a measure on $\mathbf{P}(\mathbb{N})$ and $P$ is the range of $T$. Condition (4.1) implies that $T$ is one-to-one. We turn $\mathbf{P}(\mathbb{N})$ into a metric space by defining

$$
d(A, B):=\sum_{n \in A \triangle B} 2^{-n} .
$$

It is easy to see that $T$ is continuous from $\mathbf{P}(\mathbb{N})$ onto $P$. Since $\mathbf{P}(\mathbb{N})$ is compact, this shows that $P$ is compact and $T$ is a topological map. It is also easy to see that $P$ has no isolated points and so is a perfect set. The set $P$ is called a symmetric perfect set.

The right end-points of complementary intervals of $P$ are exactly the points $T(A)$ with $A$ finite. The left end-points of complementary intervals of $P$ are exactly the points $T(A)$ with $\mathbb{N}-A$ finite.

We define $\eta_{n}:=\mu_{n} / \lambda_{n} \in(0,1)$ and $\varepsilon_{n}:=\left(1-\eta_{n}\right) /\left(1+\eta_{n}\right)$. It is easy to see that $P$ can be obtained by successively removing middle intervals from $[0,1]$ of proportion $\varepsilon_{n}$ in the $n$th step as described in [9, p. 205] and $[5$, p. 116]. The symmetric perfect set $P$ is completely determined by the numbers $\eta_{n}$ (or $\varepsilon_{n}$ ) which can be arbitrarily chosen in $(0,1)$. For example, in the Cantor set we have $\varepsilon_{n}=1 / 3, \eta_{n}=1 / 2, \lambda_{n}=2 \cdot 3^{-n}$ and $\mu_{n}=3^{-n}$.

We pose the problem: for which choices of sequences $\eta_{n}$ does $P$ belong to the class $\mathbf{P}_{k}$ ?

We present two results.

TheOREM 4.1. If $\lim \inf \eta_{n}>0$, then the symmetric perfect set $P$ is of finite Denjoy index. Thus it belongs to $\mathbf{P}_{k}$.

Pr o o f. By assumption, there is $a>0$ such that $\eta_{n} \geq a$ for all $n \in \mathbb{N}$. We claim that $P$ has finite Denjoy index with corresponding constants $\theta=\lambda_{1}$ and $\beta=2 / a$. Let $x=T(A) \in P$. We define $h_{n}:=\lambda_{n}$ if $n \notin A$ and $h_{n}:=-\lambda_{n}$ 
if $n \in A$. Then $x+h_{n} \in P$ for all $n$. Since $0<\lambda_{n} \rightarrow 0$, we have $0 \neq h_{n} \rightarrow 0$. Also, $\left|h_{1}\right|=\lambda_{1}=\theta$. Since

we obtain

$$
\frac{\lambda_{n}}{\lambda_{n+1}}=\frac{\mu_{n}}{\eta_{n} \lambda_{n+1}}=\frac{\lambda_{n+1}+\mu_{n+1}}{\eta_{n} \lambda_{n+1}}=\frac{1+\eta_{n+1}}{\eta_{n}}
$$

$$
1<\frac{\left|h_{n}\right|}{\left|h_{n+1}\right|}<\frac{2}{a}=\beta
$$

for all $n$. So $P$ has finite Denjoy index. By Corollary 3.11, $P$ belongs to $\mathbf{P}_{k}$.

Theorem 4.2. Assume that $\lim \inf \eta_{n}=0$ and $\lim \sup \eta_{n}<1$. Let $k \geq 2$. Then the symmetric perfect set $P$ does not belong to $\mathbf{P}_{k}$.

Proof. We will construct a function $f: P \rightarrow \mathbb{R}$ which is $k$ times Peano differentiable on $P$ relative to $P$ but does not admit a $k$-extension. By assumption, there is $\delta>0$ such that $1-\eta_{n} \geq \delta$ for all $n$. Moreover, there are positive integers $n_{1}<n_{2}<n_{3}<\ldots$ converging to infinity such that $\eta_{n_{i}} \rightarrow 0$. We decompose $\mathbb{N}$ into blocks $D_{i}:=\left\{n_{i-1}+1, \ldots, n_{i}\right\}, i \in \mathbb{N}$, where $n_{0}:=0$. For each subset $A$ of $\mathbb{N}$ and every $i \in \mathbb{N}$, we define $j(A, i)$ as the number of $q \in\{1, \ldots, i-1\}$ for which $A \cap D_{q}$ is nonempty. We define $f: P \rightarrow \mathbb{R}$ as follows:

$$
f(x):=\sum_{i=1}^{\infty} 2^{-j(A, i)}\left(\sum_{n \in A \cap D_{i}} \lambda_{n}\right)^{k} \quad \text { for } x=T(A) .
$$

We now show that $f$ is $k$ times Peano differentiable at a given $x \in P$ relative to $P$. We distinguish two cases:

First CASE: $x=T(A)$ and $A$ is an infinite set. Let $y=T(B) \in P$, $A \neq B$. Let $p$ be the minimal element in $A \triangle B$. Define $m$ by $p \in D_{m}$. We have

$$
|y-x| \geq \lambda_{p}-\mu_{p}=\left(1-\eta_{p}\right) \lambda_{p} \geq \delta \lambda_{p}
$$

Also,

$$
|f(y)-f(x)| \leq 2^{-j(A, m)} 2 \mu_{p-1}^{k} \leq 2^{-j(A, m)} 2^{k+1} \lambda_{p}^{k} .
$$

From (4.3) and (4.4) we obtain

$$
|f(y)-f(x)| \leq 2^{-j(A, m)} 2^{k+1} \delta^{-k}|y-x|^{k} .
$$

Now $y \rightarrow x$ implies $m \rightarrow \infty$. Since $A$ is infinite, this in turn implies $j(A, m) \rightarrow \infty$. Hence $f$ is $k$ times Peano differentiable at $x$ relative to $P$ with the first $k$ Peano derivatives equal to 0 .

SECOnd Case: $x=T(A)$ and $A$ is a finite set. Let $y=T(B), A \neq B$. Since we are only interested in $y$ close to $x$ and $A$ is finite, we can assume that $B \supset A$ so that $y>x$. Let again $p$ be the minimal element in $A \triangle B=B-A$ 
and $p \in D_{m}$. Of course, we can assume that $A \cap D_{m}=\emptyset$. Write $y-x=w+z$ with

$$
w:=\sum_{n \in B \cap D_{m}} \lambda_{n} \geq \lambda_{p} \geq \lambda_{n_{m}}
$$

and

$$
z:=\sum_{q>m} \sum_{n \in B \cap D_{q}} \lambda_{n} \leq \mu_{n_{m}}=\eta_{n_{m}} \lambda_{n_{m}} \leq \eta_{n_{m}} w .
$$

Then we have

$$
w \leq y-x=w+z \leq\left(1+\eta_{n_{m}}\right) w .
$$

This implies that

$$
0 \leq(y-x)^{k}-w^{k} \leq\left(\left(1+\eta_{n_{m}}\right)^{k}-1\right) w^{k} \leq\left(\left(1+\eta_{n_{m}}\right)^{k}-1\right)(y-x)^{k} .
$$

Setting $j:=j(A, m)=j(B, m)$ we obtain

$$
\begin{aligned}
\left|f(y)-f(x)-2^{-j} w^{k}\right| & \leq \sum_{q>m}\left(\sum_{n \in B \cap D_{q}} \lambda_{n}\right)^{k} \\
& \leq \mu_{n_{m}}^{k} \leq \eta_{n_{m}}^{k} w^{k} \leq \eta_{n_{m}}^{k}(y-x)^{k} .
\end{aligned}
$$

Thus

$$
\begin{aligned}
\left|f(y)-f(x)-2^{-j}(y-x)^{k}\right| & \leq\left|f(y)-f(x)-2^{-j} w^{k}\right|+\left|w^{k}-(y-x)^{k}\right| \\
& \leq\left\{\eta_{n_{m}}^{k}+\left(1+\eta_{n_{m}}\right)^{k}-1\right\}(y-x)^{k} .
\end{aligned}
$$

As $y \rightarrow x, j$ stays fixed but $m \rightarrow \infty$. Since $\eta_{n_{m}} \rightarrow 0$ as $m \rightarrow \infty$, we see that $f$ is $k$ times Peano differentiable at $x$ relative to $P$. The first $k-1$ derivatives are zero but the $k$ th equals $k ! 2^{-j}$.

Since the set of all $T(A)$ with finite $A$ is dense in $P$, Corollary 3.10 shows that $f$ does not admit a $k$-extension. So $P$ does not belong to $\mathbf{P}_{k}$.

Theorems 4.1 and 4.2 solve our problem except in the case of

$$
\liminf \eta_{n}=0 \quad \text { and } \quad \lim \sup \eta_{n}=1 \text {. }
$$

This leads us to asking the question: can a symmetric perfect set $P$ whose corresponding sequence $\eta_{n}$ satisfies (4.5) belong to $\mathbf{P}_{k}$ ?

\section{References}

[1] Z. Buczolich, Second Peano derivatives are not extendable, Real Anal. Exchange 14 (1988-89), 423-428.

[2] Z. Buczolich and C. Weil, Extending Peano differentiable functions, Atti Sem. Mat. Fis. Univ. Modena 44 (1996), 323-330.

[3] P. Bullen, Denjoy's index and porosity, Real Anal. Exchange 10 (1984-85), 85-144.

[4] A. Denjoy, Sur l'intégration des coefficients différentiels d'ordre supérieur, Fund. Math. 25 (1935), 273-326. 
[5] A. Denjoy, Leçons sur le calcul de coefficients d'une série trigonométrique I-IV, Gauthier-Villars, Paris, 1941-1949.

[6] H. Fejzić, The Peano derivatives, doct. dissertation, Michigan State Univ., 1992.

[7] H. Fejzić, J. Mařík and C. Weil, Extending Peano derivatives, Math. Bohem. 119 (1994), 387-406.

[8] H. W. Oliver, The exact Peano derivative, Trans. Amer. Math. Soc. 76 (1954), $444-456$.

[9] B. Thomson, Real Functions, Lecture Notes in Math. 1170, Springer, Berlin, 1985.

Department of Mathematical Sciences

University of Wisconsin-Milwaukee

P.O. Box 413

Milwaukee, Wisconsin 53201

U.S.A.

E-mail: volkmer@csd.uwm.edu

Received 18 June 1997;

in revised form 2 February 1998 and 24 September 1998 